\title{
Spinal Neoplasm in Dogs
}

\author{
Larissa Garbelini Valentim ', Rogério Anderson Marcasso², \\ Ana Paula Frederico Loureiro Bracarense ${ }^{3}$ \& Mônica Vicky Bahr Arias' ${ }^{1}$
}

\begin{abstract}
Background: Various diseases can cause spinal cord disorders in dogs including neoplasia of the nervous system, which are more common in mature and older animals. Some breeds, as brachycephalics, are more predisposed for certain types of tumors. Spinal neoplasia can be categorized as primary or secondary tumors, and still can be grouped in extradural, intradural/extramedullary or intramedullary. The aim of this study was to investigate the signalment, neurological syndrome, type and source of the mass, presence of metastases, and clinical outcomes in 28 dogs diagnosed with spinal tumors.

Materials, Methods \& Results: This study included 28 dogs, and in 20 cases, spinal neoplasia was confirmed by necropsy, biopsy, histopathology, and immunohistochemistry. In the other eight animals, the presumptive diagnosis was based on clinical and neurological signs and by observing changes in plain radiographs of the spine, chest radiographs, and myelography. The location of the lesion was established as cervical, cervicothoracic, thoracolumbar, or lumbosacral, and the lesion was determined to be multifocal when clinical signs appeared in more than one location. Spinal cord injury was additionally classified as unilateral, bilateral, symmetrical, or asymmetrical. The following complementary examinations were performed based on the clinical suspicion, indications, and availability of the owner: complete blood count, serum biochemical profile, urinalysis, spinal radiography, cerebrospinal fluid analysis (CSF), myelography, abdominal ultrasound, thoracic X-ray to determine metastases, and Computed Tomography. CSF was collected and analyzed in seven animals, and pleocytosis with a predominance of lymphocytes was found in two cases. Decompression and exploratory surgeries were performed in six dogs to confirm the suspected diagnosis and collect tissues for histopathology. The final diagnosis was made by histopathological examination of the tissue obtained by excisional biopsy or necropsy after the death of the animal or euthanasia. In eight cases, the neoplasia type could not be confirmed as the owner did not provide consent to perform exploratory surgery or necropsy. The dogs most affected were poodles (17.85\%) and boxers (17.85\%), between the ages of 5 and 10 years, and $67.85 \%$ of the cases were female dogs. The thoracolumbar region was the most affected (50\%). Metastatic spinal tumors predominated; especially those originating in the mammary gland (38.46\%) and on the skin (34.61\%), and among the primary neoplasms, meningiomas predominated. The clinical course in relation to the onset of symptoms of neurological dysfunction, until death or euthanasia, ranged from 1-170 days, with an average 42 days of survival.

Discussion: The results differ from previous studies, in which the number of male and female dogs with neoplasms in the CNS was the same, as in the present study female dogs were more affected. Unlike other studies, metastatic spinal tumors predominated. After the diagnosis, the survival was short and this may be due to the fact that most dogs in this study already had more than one system affected, and consequently showed significant changes related to systemic metastases during the onset of the neurological symptoms. The surgical treatment presented variable results, and the results observed after surgical resection of neoplasms vary due to the location in relation to the spinal cord, neoplasm type, severity of the clinical signs, and possibility of removal along with margin without causing spinal instability. In dogs with spinal disorders, the veterinary practitioner should suspect cancer, especially if there is a history of excision of neoplasms in other systems.
\end{abstract}

Keywords: spinal cord, nervous system, neoplasms, dogs. 


\section{INTRODUCTION}

Various diseases can cause spinal cord disorders in dogs [5], including neoplasia [7]. Dogs are one of the domestic species most affected by tumors in the central nervous system $[9,11,16]$. Spinal tumors can be categorized as primary or secondary, and they can be grouped as tumors arising from an extradural location (extradural), situated within the dura but outside the spinal cord (intradural/extramedullary), or arising within the spinal cord parenchyma (intramedullary) [5]. Extradural tumors are the most common spinal tumors observed in dogs, and include primary and secondary bone tumors $[11,15,16]$.

Neurological signs resulting from spinal cord impairment depend on different factors, such as the spinal region affected, location of the mass in relation to the spinal cord, cell type, malignancy, growth rate, compression rate, destruction, edema, hemorrhage, and spinal cord adaptability $[7,13,17]$. The presumptive diagnosis can be made from clinical and neurological evaluations associated with radiography, myelography, computed tomography or magnetic resonance imaging. A definitive diagnosis is made only after performing histopathology by either biopsy or necropsy $[6,11,14]$.

The aim of this study was to evaluate the information obtained from a review of the medical history, physical and neurological examination, complementary test results, type and origin of the mass, presence of metastases, and clinical outcomes in dogs with spinal neoplasms treated at the Veterinary Hospital (HV) from Universidade Estadual de Londrina (UEL), between January 2007 and May 2013.

\section{MATERIALS AND METHODS}

The records of dogs treated at the HV/ UEL between January 2007 and May 2013 with a final diagnosis of spinal neoplasm were assessed. The following data were evaluated: breed, sex, age, reproductive status, history of cancer in another region, age at onset, and evolution time of clinical symptoms. During the physical examination, cardiac and respiratory frequencies, rectal temperature, degree of hydration, color of mucous membranes, femoral pulse and size of abdominal, rectal, and superficial lymph node were recorded. The neurological examination included evaluation of postural reactions, cranial nerves, reflexes, nociception and presence of vertebral hyperpathia. The location of the lesion was established as cervical, cervicothoracic, thoracolumbar, or lumbosacral, and the lesion was determined to be multifocal when clinical signs appeared in more than one location. Spinal cord injury was additionally classified as unilateral, bilateral, symmetrical, or asymmetrical [17].

The following complementary examinations were performed based on the hypothesis, indications, and availability of the owner: complete blood count, serum biochemical profile, urinalysis, spinal radiography, cerebrospinal fluid analysis, myelography, abdominal ultrasound, X-ray to determine thoracic metastases and CT. Myelography was performed by lumbar or cisternal injection of iohexol ${ }^{1}$. Decompression and exploratory surgeries were also performed in six dogs to confirm the suspected diagnosis and collect tissues for neoplasm histopathology.

The final diagnosis was made by histology from samples obtained by excisional biopsy or necropsy after the death of the animal or euthanasia. In some cases, the neoplasia type could not be confirmed as the owner did not provide consent to perform exploratory surgery or necropsy. Neoplasm sections were fixed in $10 \%$ buffered formalin, processed routinely, and 5-mm thick sections were stained with hematoxylin-eosin (HE).

In six cases, an immunohistochemical examination was performed to obtain a definitive diagnosis and histological classification of the neoplasm. Histological sections in previously silanized slides were deparaffinized and subjected to antigen retrieval in a citrate buffer solution ( $\mathrm{pH}$ of 6.0) in a microwave oven, followed by blocking of endogenous peroxidase and incubation with primary antibodies, such as anti-pan cytokeratin $^{2}$; anti-S100 protein ${ }^{3}$; anti-glial fibrillary acidic protein ${ }^{3}$, anti-VIII-related antigen factor4; and anti-Vimentin V9 clone ${ }^{4}$, at 1:100 dilutions in a humid chamber refrigerated for $18 \mathrm{~h}$. The sections were then incubated with secondary antibodies for $30 \mathrm{~min}$ in a $\mathrm{T}^{\circ} \mathrm{C}$ environment, and the samples were identified using chromogen $\mathrm{DAB}^{\circledR}$ solution ${ }^{3}$. The samples were counterstained with Harris hematoxylin, followed by slide assembly with commercial resin. Positive and negative controls were used in all reactions (small intestine for anti-PCK; cerebral cortex for anti-S100 and anti-GFAP; lung for anti-FVIII and muscle for anti-Vin).

The dogs were grouped into the following groups: Group A, conclusive diagnosis, confirmed spinal neoplasm (20 dogs) and Group B, presumptive diagnosis ( $8 \mathrm{dogs})$. 


\section{RESULTS}

Twenty-eight dogs (19 females and 9 males) were examined during the study period (Table 1 ). The age of the dogs ranged from 2.5 to 17 years, with a mean age of 9.5 years. The weight of the animals ranges from 2.7 to $37 \mathrm{~kg}$ and $15 \mathrm{dogs}(53.57 \%)$ weighed above $20 \mathrm{~kg}$. The most commonly affected breeds were Mongrel dogs (6/28), Poodle (5/28), Boxer (5/28), Rottweiler (2/28), Dalmatian (2/28), Cocker spaniel (2/28), Labrador (1/28), Australian cattle dog (1/28), Doberman (1/28), Fila brasileiro (1/28), Pinscher $(1 / 28)$, and Bull terrier $(1 / 28)$.

Table 1. Breed, gender, age, localization, neurological syndrome in dogs with spinal neoplasms (Group A) and dogs with a presumptive diagnosis of spinal neoplasm (Group B) from 2007 to 2013. Veterinary Hospital (HV), Universidade Estadual de Londrina (UEL), Paraná State, Brazil.

\begin{tabular}{|c|c|c|}
\hline & Group A & Group B \\
\hline Breed & $(\mathrm{n}=20)$ & $(\mathrm{n}=8)$ \\
\hline Mongrel & 6 & 0 \\
\hline Boxer & 3 & 2 \\
\hline Poodle & 3 & 2 \\
\hline Dalmatian & 2 & 0 \\
\hline Labrador & 1 & 0 \\
\hline Australian Cattle Dog & 1 & 0 \\
\hline English Cocker Spaniel & 1 & 1 \\
\hline Rottweiler & 1 & 1 \\
\hline Doberman & 1 & 0 \\
\hline Pinscher & 0 & 1 \\
\hline Brazilian Mastiff & 0 & 1 \\
\hline Bull Terrier & 1 & 0 \\
\hline \multicolumn{3}{|l|}{ Sex } \\
\hline Female & 13 & 7 \\
\hline Male & 7 & 1 \\
\hline \multicolumn{3}{|l|}{ Age (years) } \\
\hline$<5$ & 2 & 0 \\
\hline $5-10$ & 13 & 5 \\
\hline $11-15$ & 4 & 3 \\
\hline$>15$ & 1 & 0 \\
\hline \multicolumn{3}{|l|}{ Alteration } \\
\hline Ataxia & 2 & 1 \\
\hline Paraparesis & 6 & 1 \\
\hline Paraplegia & 7 & 5 \\
\hline Tetraparesis & 0 & 1 \\
\hline Tetraplegia & 4 & 0 \\
\hline \multicolumn{3}{|l|}{ Neurological Syndrome } \\
\hline Cervical & 3 & 1 \\
\hline Cervicothoracic & 0 & 0 \\
\hline Thoracolumbar & 8 & 5 \\
\hline Lumbosacral & 5 & 2 \\
\hline Multifocal & 4 & 0 \\
\hline \multicolumn{3}{|l|}{$\begin{array}{c}\text { Previous surgery for nodule } \\
\text { removal }\end{array}$} \\
\hline Mammary & 2 & 2 \\
\hline Cutaneous & 0 & 2 \\
\hline
\end{tabular}

Table 2. Spinal radiography, myelography, cerebrospinal fluid (CSF), presence of metastases, decompressive surgery performed, and final outcome in animals with confirmed diagnosis of spinal neoplasms (Group A) and in animals with presumptive diagnosis of spinal neoplasms (Group B) from 2007 to 2013. Veterinary Hospital (HV), Universidade Estadual de Londrina (UEL), Paraná State, Brazil.

\begin{tabular}{|c|c|c|}
\hline & Group A & Group B \\
\hline $\begin{array}{l}\text { Changes on the spinal } \\
\text { radiography }\end{array}$ & $(\mathrm{n}=20)$ & $(\mathrm{n}=8)$ \\
\hline Yes & 8 & 6 \\
\hline No & 9 & 0 \\
\hline Not performed & 3 & 2 \\
\hline \multicolumn{3}{|l|}{$\begin{array}{l}\text { Changes on the } \\
\text { myelography }\end{array}$} \\
\hline Yes & 8 & 3 \\
\hline No & 0 & 0 \\
\hline Not performed & 12 & 5 \\
\hline \multicolumn{3}{|l|}{ Changes in the CSF } \\
\hline Yes & 2 & 0 \\
\hline No & 3 & 3 \\
\hline Not performed & 15 & 5 \\
\hline \multicolumn{3}{|l|}{ Neoplasms in other organs } \\
\hline One organ & 4 & 4 \\
\hline More than one organ & 9 & 2 \\
\hline Marrow only & 7 & 2 \\
\hline Mammary glands & 7 & 4 \\
\hline Cutaneous & 8 & 2 \\
\hline Kidneys & 3 & 1 \\
\hline Liver & 4 & 0 \\
\hline Spleen & 4 & 0 \\
\hline Tonsils & 1 & 0 \\
\hline Cerebrum & 2 & 0 \\
\hline Lungs & 3 & 2 \\
\hline Stomach & 1 & 0 \\
\hline Intestine & 2 & 0 \\
\hline Skull & 1 & 0 \\
\hline Abdominal Wall & 1 & 0 \\
\hline Chest Wall & 1 & 0 \\
\hline Heart & 1 & 0 \\
\hline \multicolumn{3}{|l|}{ Decompressive surgery } \\
\hline Dorsal Laminectomy & 2 & 0 \\
\hline Hemilaminectomy & 4 & 0 \\
\hline Not performed & 14 & 8 \\
\hline \multicolumn{3}{|l|}{ Final outcome } \\
\hline Euthanasia & 15 & 5 \\
\hline Death & 3 & 0 \\
\hline $\begin{array}{c}\text { Palliative Treatment or } \\
\text { survival }\end{array}$ & 2 & 3 \\
\hline
\end{tabular}

In Group A, primary neoplasms were observed in seven cases (35\% [7/20]) and metastatic neoplasms were observed in 13 (65\% [14/20]). Twelve animals had masses in more than two organs, seven had masses in more than one organ besides the spinal cord, and nine showed no metastases in other systems. The location of the spinal neoplasms and metastasis are shown in Table 2; the mammary gland and skin neoplasms were the most frequent source of metastasis to the nervous 
system. In Group B, the diagnosis was made by record of prior skin and/or mammary tumor excision, the typical changes observed in the spinal radiographs, and/ or myelography or presence of pulmonary metastasis on chest radiography, associated with the presence of tumors on other systems, and in six out of eight dogs the prime suspect was secondary neoplasm.

The spinal syndromes observed were cervical (3/28), cervicothoracic (1/28), thoracolumbar (13/28), lumbosacral (7/28), and multifocal (4/28) syndromes. However, out of the 14 cases with thoracolumbar syndrome, neoplasms were found in the cranial thoracic vertebrae in four. Two were meningiomas, one a transmissible venereal tumor, and one a lymphoma.

Spinal radiography was performed in 23 animals. Fourteen animals (61\% [14/23]) showed various changes, including bone lysis (10/14), bone proliferation (4/14), increase in the spinal canal diameter (1/14), change in vertebra shape (3/14), irregularity and reduced intervertebral space (3/14), and vertebral body fracture with reduced radiopacity in the vertebral body (1/14) [Figure 2]. Myelography was performed in 11 animals $(11 / 28)$, and in all cases, an interruption or deviation of the column of contrast (Figure 3) was observed. One of these dogs, a Boxer, had a myelotomography performed, because in the myelography there was interruption of the contrast, not being possible to perform a diagnosis. The CT myelography suggested the presence of an intradural-extramedullary mass. The alterations found is detailed in Table 3. Out of 20 dogs with a confirmed diagnosis of spinal neoplasms, 10 had tumors in extradural locations $(50 \%$ $[10 / 20])$, six in intradural-extramedullary locations $(30 \%[6 / 20])$, and four in intramedullary locations (20\% [4/20]). Out of the four cases of intramedullary neoplasms, one was primary (meningioma) and found in the lumbosacral region of a 12-year-old dog [1/4], while three were multifocal, of which one was found in the cervical and thoracolumbar region of a 7-yearold dog with lymphoma, one was a hemangiosarcoma in a 17-year-old dog, and one 2.5 year-old dog had a malignant histiocytosis.

Cerebrospinal fluid was collected and analyzed in seven animals, and pleocytosis with a predominance of lymphocytes was found in two, and in these cases the final histopathologic diagnosis was spinal lymphoma. In one of these patients, a Bull Terrier, in addition to the presence of thoracic, abdominal, pleural, and pericardial effusion, it was observed metastasis in the heart, kidney, mammary gland, and mesentery, and neurological symptoms developed over a period of 17 days. No significant results were obtained from the five other cerebrospinal fluid examination.

Table 3. Changes observed on myelography and spinal radiography in dogs with a confirmed diagnosis (Group A) and presumptive diagnosis (group B) of spinal neoplasia from 2007 to 2013 . Veterinary Hospital (HV), Universidade Estadual de Londrina (UEL), Paraná State, Brazil.

\begin{tabular}{cc}
\hline & Group A, B \\
\hline Changes found in the myelography & $(\mathrm{n}=11)$ \\
\hline Interruption of column of contrast & 8 \\
Asymmetry of column of contrast & 1 \\
Dorsal deviation of column of & 4 \\
contrast & $(\mathrm{n}=14)$ \\
Changes found on the spinal & 10 \\
radiography & 4 \\
Bone lysis & 1 \\
Bone proliferation & 3 \\
Increase in spinal canal diameter & 3 \\
Change in the vertebra structure & \\
Irregularity and decreased & 1 \\
intervertebral space & \\
Pathological fracture of the vertebral & body
\end{tabular}

Six animals (6/28) underwent decompression surgery; two were dorsal laminectomies (2/6) and four were hemilaminectomies (4/6). Three animals were euthanized during the perioperative period. In the Boxer dog with a mass diagnosed by myelotomography, during the dorsal laminectomy, a transoperative imprint was performed, with an initial result of meningioma, and due to infiltration of the mass in the spinal cord, the animal was euthanized (Figure 3). In a mongrel dog presenting a lumbosacral syndrome, radiography revealed an increase in the size of the spinal canal at L5 (Figure 2), while myelography revealed an interrupted contrast spine between L4 and L5. The patient underwent an exploratory dorsal laminectomy, in which was observed an irregular increase of the spinal cord volume, causing spinal cord impairment. Thus, the dog was euthanized in the perioperative period. Histopathological examination of this mass showed grade III anaplastic meningioma. A poodle with meningothelial meningioma of the T2 vertebra (Figure $3 \mathrm{C}$ and D) showed initial improvement after $\mathrm{T} 2$ surgical decompression associated with chemotherapy with doxorubicin, and was able to walk again. The chemotherapy was performed because the initial results of histopathological examination sug- 
gested sarcoma. After the third chemotherapy session with doxorubicin, the general condition of the animal deteriorated, the owner opted for euthanasia, being the total survival time 85 days after the surgery. A paraplegic Dalmatian due to a meningioma in T2-T4 showed good postoperative recovery after total removal of the tumor. The dog started to walk with ataxia using a wheeled cart, and was accompanied until day 135 , showing good recovery without recurrence. Another Dalmatian was diagnosed with extradural metastasis of a transmissible venereal tumor (TVT) in vertebra T8, showing significant improvement after surgical removal of the mass. Surgical treatment was then associated with chemotherapy with vincristine and the dog is now able to walk with mild spinal ataxia and has shown no recurrence of the condition.

Out of the primary neoplasms found, seven were meningiomas and affected mainly male dogs. Myelography was performed in six cases. In one of these cases the result was inconclusive and the case was referred to myelotomography. In the other five dogs, changes were compatible with extradural lesion in three cases, intradural-extramedullary in one and intramedullary in one. In one dog the lesion was in the cervical region, in two they were in the cervicothoracic region, in three in the thoracolumbar region, and in one in the lumbosacral region. In the latter patient a simple radiograph was performed previously and it was observed expansion of the spinal canal with change in the dorsal laminae of the spinal canal of the L5 vertebra. In another patient probably due to infiltration mass on $\mathrm{T} 1$ vertebra, it was observed in the plain radiograph pathological fracture of the vertebral body.

Regarding secondary malignancies, three dogs had lymphoma (3/20) and two of them had generalized lymphadenopathy and increased number of lymphocytes in the CSF. Hemangiosarcoma was diagnosed in one dog presenting paraplegia and seizures, being the primary neoplasm originated in the skin, with metastases in the liver, spleen, mammary gland, and brain (Figure $1 \mathrm{~F}, \mathrm{G}$, and $\mathrm{H}$ ). However, in this dog, metastasis of the hemangiosarcoma was intramedullary. One dog had osteosarcoma in the L7 and sacral (1/20) vertebrae. Proliferation in the L7 and sacral vertebrae as well as bone destruction was observed on the radiography.

Two dogs (2/20) presented malignant histiocytosis (Figure 1A, B, and C), and both showed splenomegaly and hepatomegaly. One presented dyspnea and paraplegia, while the other tetraplegia and lymphadenopathy. In one dog, the final diagnosis was spinal metastasis of intestinal tubular adenocarcinoma, with tumor emboli, which resulted in a malacia area on the site, and this animal also presented metastasis in the mammary gland, lung, brain, liver, and spleen. Two (2/20) animals presented diagnoses compatible with mammary tumor. Although both had a history of mammary tumor, it was not possible, however, to classify the type of carcinomas, which were located in the extradural lumbar region. The frequency of primary and metastatic spinal tumors are summarized in Table 4.

Table 4. Neoplasm localization and types of neoplasms found in animals with confirmed diagnosis of spinal neoplasms (Group A) from 2007 to 2013. Veterinary Hospital (HV), Universidade Estadual de Londrina (UEL), Paraná State, Brazil.

\begin{tabular}{cc}
\hline & Group A \\
\hline Localization of neoplasm & $(\mathrm{n}=20)$ \\
\hline Extradural & 10 \\
Intradural-extramedullary & 6 \\
Intramedullary & 4 \\
\hline Types of neoplasm & \\
\hline Meningioma & 7 \\
Lymphoma & 3 \\
Malignant histiocytosis & 2 \\
Micropapillary carcinoma & 1 \\
Hemangiosarcoma & 1 \\
Multiple myeloma & 1 \\
Transmissible venereal tumor & 1 \\
Osteosarcoma & 1 \\
Intestinal tubular adenocarcinoma & 1 \\
Mammary carcinoma & 2 \\
\hline
\end{tabular}

In eight animals (8/28), the diagnosis of spinal tumor was presumptive and based on the results of laboratory tests, radiography (5/8), myelography (3/8), chest radiography with pulmonary metastasis $(3 / 8)$, record of prior skin and/or mammary tumor excision (3/8), and the presence of nodules in the body on clinical examination (4/8) [Figure 4].

The clinical course in relation to the onset of symptoms of neurological dysfunction, until death or euthanasia, ranged from 1-170 days, with an average 42 days of survival. Twenty animals were euthanized, three died, and three underwent palliative treatment, which included rest, nursing care, bladder massage, and use of analgesics and anti-inflammatory medications. Only two dogs survived, one with canine transmissible venereal tumor and one with meningioma, both treated with surgery. 


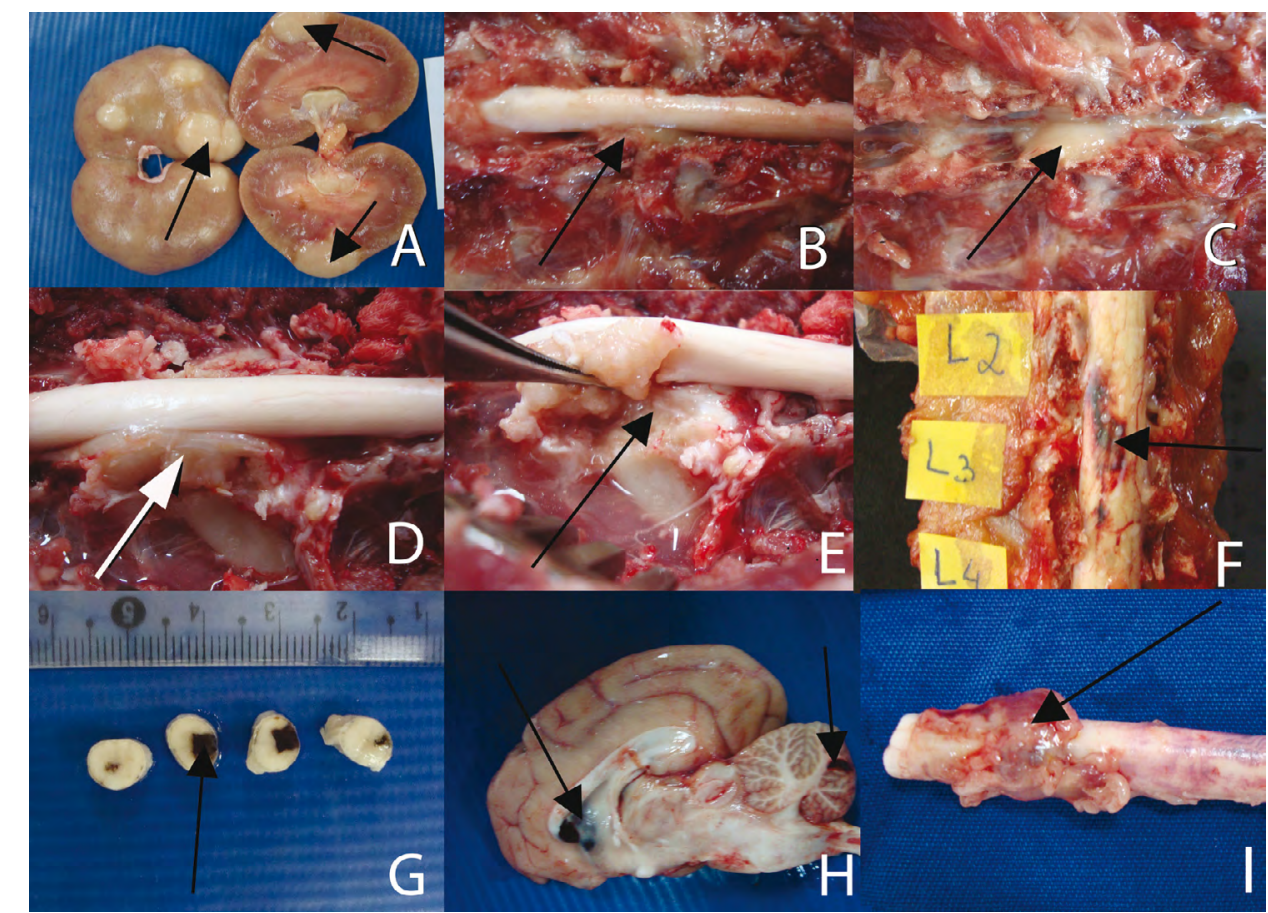

Figure 1. Images of necropsy in dogs with spinal neoplasms. A- Malignant histiocytosis in dogs with paraplegia due to thoracolumbar syndrome and kidney metastasis (arrows). B- Same dog, presence of mass in the lumbar vertebra L2 (arrow). C- Same dog, mass in the spinal canal floor after removal of the spinal cord (arrow). DMeningioma in the vertebra L5 of a Cocker Spaniel with paraplegia due to lumbosacral syndrome. Presence of a mass compressing the spinal cord (arrows). E- Same dog, infiltration of the mass into the vertebral body (arrow). F- Medullary segments L2, L3, and L4 of a female mutt with paraplegia due to an intramedullary hemangiosarcoma (arrow). G- Same dog, neoplasia is intramedullary. H- Same dog, cerebrum metastasis (arrows). I- Lymphoma metastasis in spinal segment $\mathrm{T} 7$ of a female Bull Terrier with paraplegia.
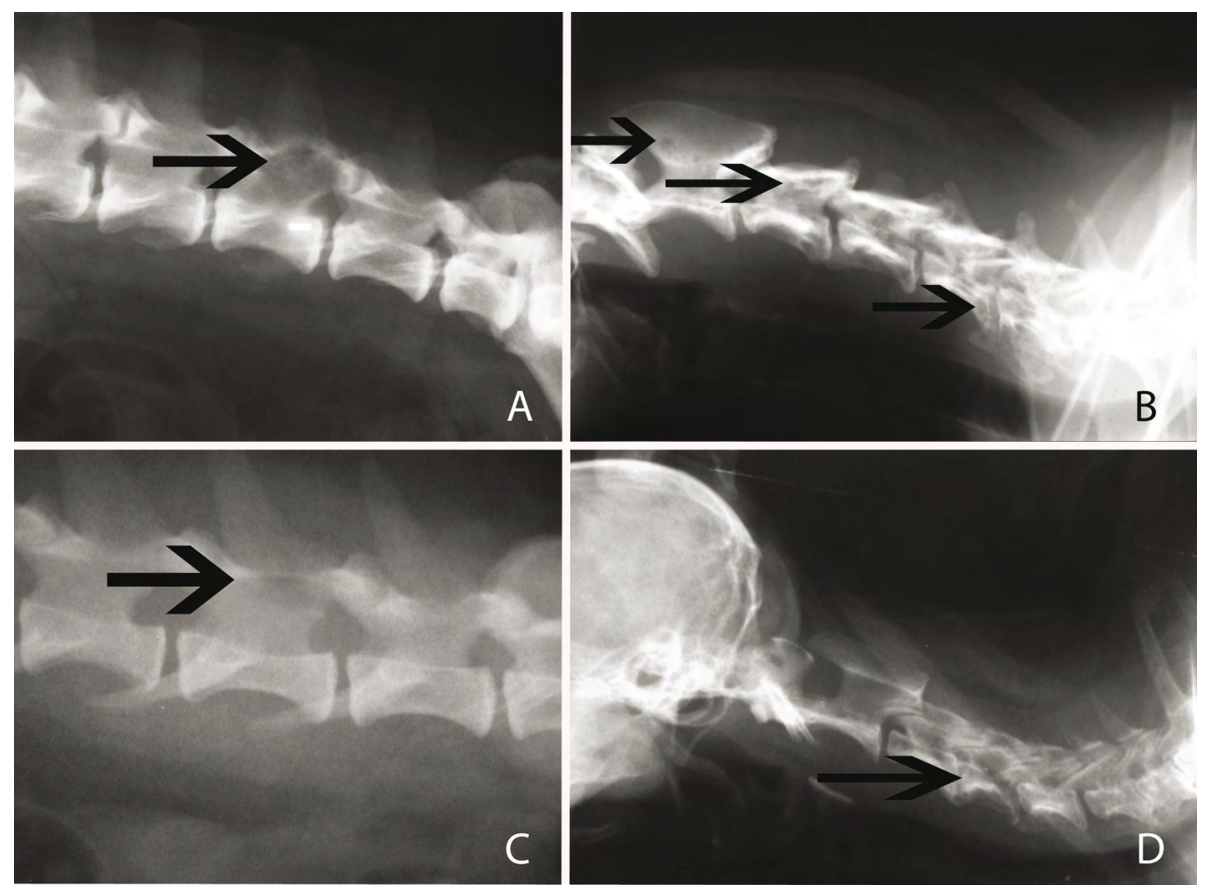

Figure 2. Radiographs of dogs with spinal neoplasms. A- Bone lysis in the L5 vertebra lamina of a 10-yearold Boxer presenting progressive paraparesis. The arrow points to the edge of the lesion. B- Several lytic, piecemeal lesions in the cervical vertebrae of a dog diagnosed with multiple myeloma. Arrows indicate some of the lesions. C- Increased size of the medullary canal in the L5 vertebra due to lysis of dorsal laminae due to an intramedullary tumor growth (meningioma). The arrow points to the cranial edge of the lesion. D- Deformity of the caudal border of the body of the $\mathrm{C} 4$ vertebra in a 15-year-old Pinscher presenting tetraparesia. The arrow points to the normal cranial edge of the vertebra. It is possible to see lysis in the middle third of the $\mathrm{C} 4$ vertebra in comparison with the normal structure of the neighboring vertebrae. 

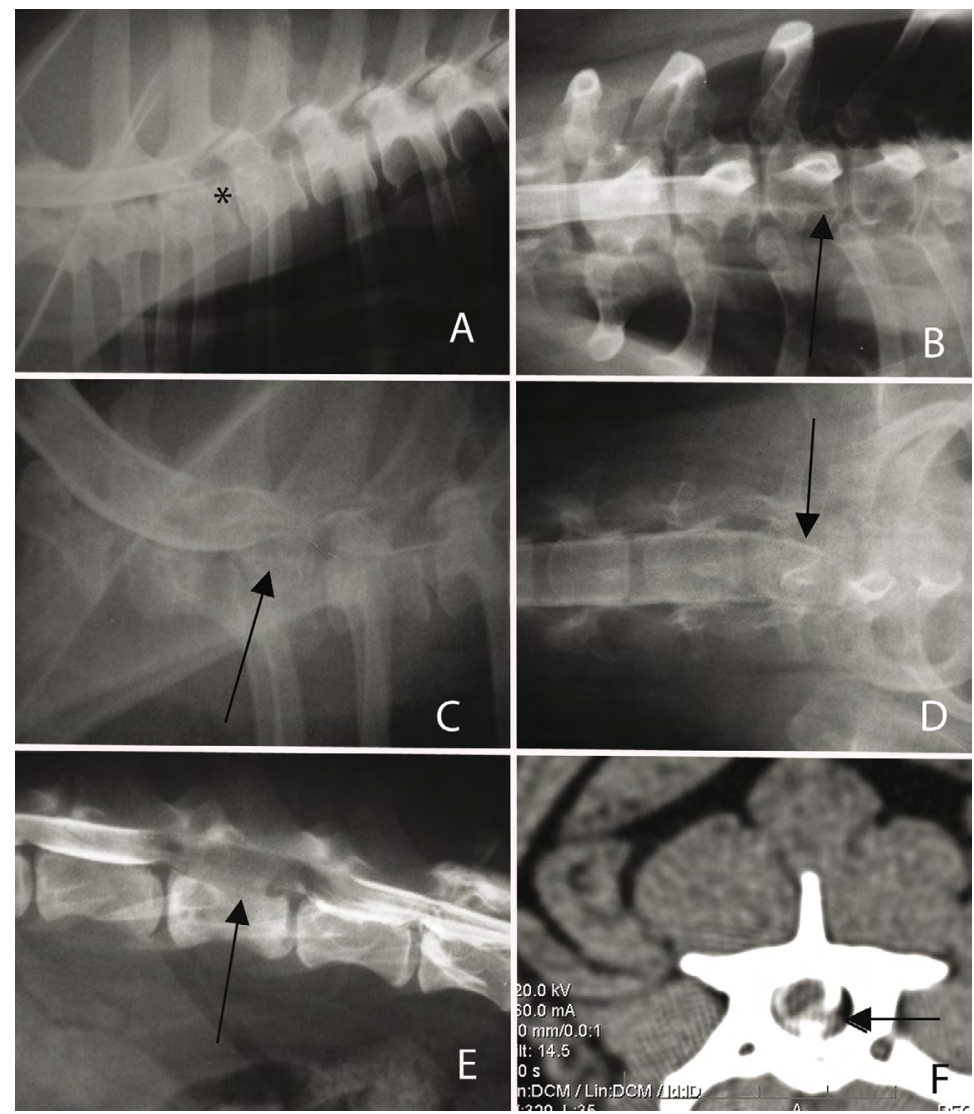

Figure 3. Photograph of diagnostic imaging tests performed in dogs with spinal neoplasms. A- Myelography of a Dalmatian with meningioma in the T3 vertebrae, lateral radiograph: interruption of the column of contrast is observed over the T3 vertebrae. B- Myelography of a Dalmatian with meningioma in the T3 vertebrae, ventrodorsal radiograph: asymmetry in the left side (arrow) in which the column of contrast can be seen, and the right side, which lacks of contrast. C- myelography of a Poodle with meningioma of the T1 vertebra. On the lateral projection, a deviation of the column of contrast on T1 and decreased body size of the T1 vertebra are observed (arrow). D- Myelography of a Poodle with meningioma of the T1 vertebra; ventrodorsal projection: a deviation of the column of contrast is observed (arrow).E- Myelography of a Cocker Spaniel. Deviation and interruption of the column of contrast over the vertebral body L4 are observed (arrow). F- Transverse myelotomography of a Boxer with meningioma of the C4 vertebrae on the left side (arrow).

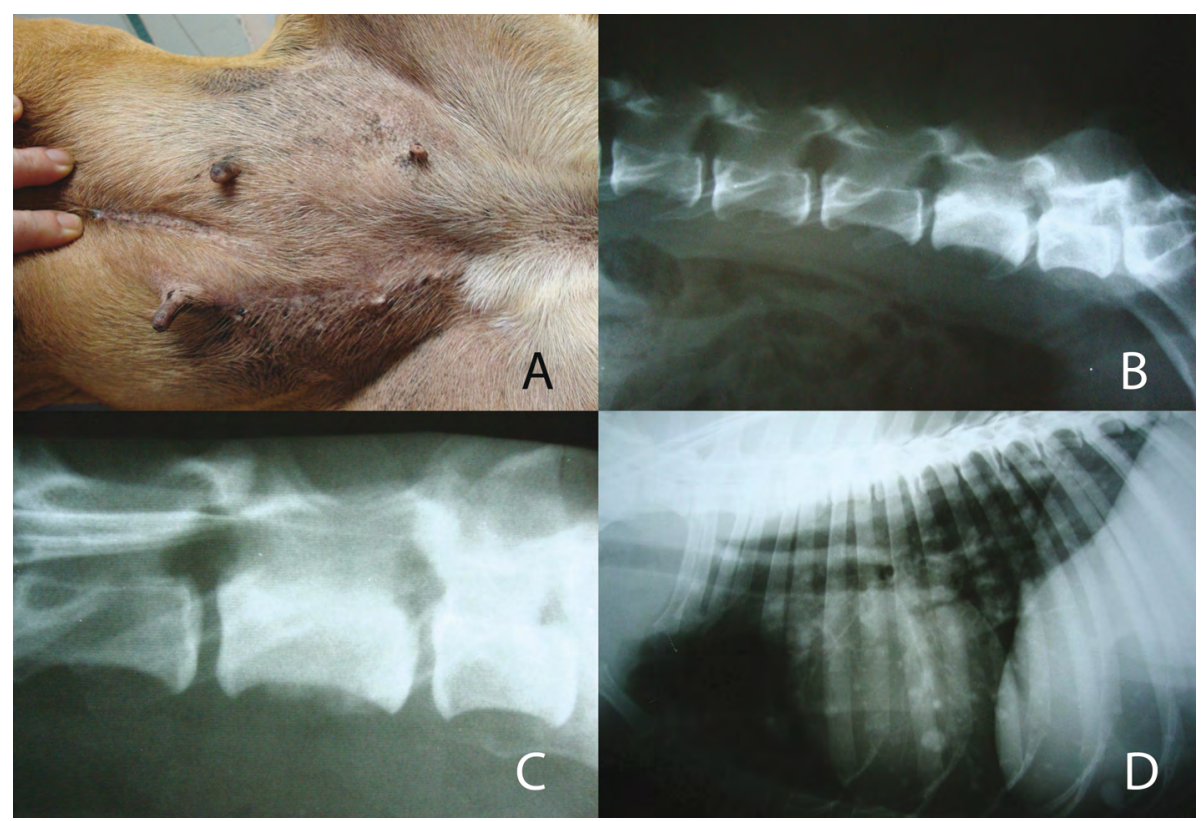

Figure 4. Photographs of a female Boxer with a presumptive diagnosis of spinal neoplasm. A- Scar of a regional mastectomy performed seven months before. B- Lumbosacral radiography, with increased bone radiopacity in L6-L7. C- Myelography, with interruption of the column of contrast over L5-L6 and L7, suggesting the presence of a mass inside the vertebral canal. D- Thoracic radiograph, with the presence of several diffuse nodules (arrows) in the lung fields, which are suggestive of pulmonary metastases. 


\section{DISCUSSION}

The results differ from previous studies, in which the number of male and female dogs with neoplasms in the CNS was the same [5,14], as in the present study female dogs were more affected. Unlike other studies [14,16], metastatic spinal tumors predominated. The mean age of the affected dogs was similar to that of the dogs in another study in Brazil [16]. As in other studies, boxers $[13,14]$ and large dogs $[6,9,11]$ were the most affected by spinal tumors, however poodle was a common breed too, probably because of a greater preference for these breed in the region of the country in which this study was conducted.

The neurological syndrome most often encountered was thoracolumbar, as described in other researches $[9,11,14]$. However, out of the 14 cases with thoracolumbar syndrome, neoplasms were found in the cranial thoracic vertebrae in four. Two were meningiomas, one a transmissible venereal tumor, and one a lymphoma. This rostral localization is in agreement with previous information that stated that the thoracolumbar region of the cranial thoracic region is most prone to neoplasms [9].

The findings observed in plain radiographs were also reported in other studies $[9,11,15]$, who state that bone lysis, bone proliferation, increase in the spinal canal diameter as a result of spinal tumor expansion and pathological fracture in the body or vertebral lamina, are common findings in patients with tumors that affect the vertebrae. These findings were evident in $61 \%$ of survey radiographs performed, representing $50 \%$ of the cases, so this technique can provide many important diagnostic information.

Although advanced image as magnetic resonance or tomography are superior to myelography for the diagnosis [5], these tests are not available in the area where the present study was done, therefore myelography was very useful. Myelographic alterations as interruption or deviation of the contrast column indicated localization of the neoplasm [17] in ten out of 11 cases, and the three basic pathologic patterns were recognized, being the frequency of extradural, intradural-extramedullary and intramedullary lesions similar to previous publications $[9,15]$, that stated that $50 \%$ of tumors are extradural, $35 \%$ are intradural-extramedullary, and $15 \%$ intramedullary. These findings can be accurate at locating the tumor along the neuraxis, especially when the tumor is a meningioma [15].
Examination of cerebrospinal fluid may be normal in dogs with spinal neoplasia [17], as observed in the present study, as most of CSF were normal. Sometimes there is an increase in the protein concentration without an increase in the number of cells, called albuminocytologic dissociation, but this was not observed [17]. The most common tumor associated with neoplastic cells present in the CSF is lymphosarcoma [17], as observed in the present study, since there was predominance of lymphocytes in two cases however, the morphology of the cells were not evaluated.

Out of the primary neoplasms found, seven were meningiomas, one in the cervical region, two in the cervicothoracic region, three in the thoracolumbar region, and one in the lumbosacral region. Meningioma is the most frequent primary CNS neoplasm affecting the spinal cord in dogs [15], and in the present study, it was the most common neoplasm after the secondary ones. The prevalence of thoracolumbar localization was different from many previous studies that found a higher frequency of meningiomas in the cervical region $[2,13]$.

Despite the present study have found more females than males with spinal neoplasia, most of the patients with meningiomas were males. There are different results between studies about gender predominance in dogs with meningioma [2] and in some studies there is a male:female predominance of $2: 1$. In other studies there are more females than males with meningiomas [1]. Data from human and veterinary medicine suggest that the growth of meningiomas may be influenced by the female sex hormones [1].

The prognosis for spinal meningiomas depends on the location, degree of invasion of the nervous tissue, and severity of neurological symptoms during diagnosis [4]. Surgery may permit complete removal or cytoreduction and biopsy of the tumor, however, removing spinal meningiomas is more problematic than excising intracranial meningiomas, because they tend to be more infiltrative [2], as observed in some cases, in which tumor excision was not possible.

Despite lymphoma being one of the most common neoplasms in the dog [3], spinal lymphoma is more common in cats than in dogs $[5,6]$. However, in the present study there were three cases of lymphoma. This disease can involve any tissue outside of the lymphatic system including the central nervous [3]. CSF changes not always occur in dogs with lymphoma 
$[6,9,15]$, but in two cases there was CSF alterations. In a study with six dogs with spinal lymphoma, it was observed that this disease is multifocal, causing vertebral involvement, spinal cord compression and involvement of more than one spinal compartment, like medullary cavity, vertebral canal and paraspinal soft tissues [3].

System malignant histiocytosis, diagnosed in two animals, is an aggressive, multisystemic disease characterized by multiple tumors in several organs, with the spleen, lung, and bone marrow involved primarily and the liver and lymph nodes secondarily, as seen in the present study [8]. Neurologic signs are reported in $38 \%$ of dogs with malignant histiocytosis, and diagnosis is a challenge for the clinician because affected dogs often have nonspecific clinical signs that indicate multisystem involvement, as seen in the present study [8].

Many kinds of carcinomas can metastasize to the vertebrae via the haematogenous route including mammary carcinoma [17], as seen in the present study. Carcinomas are often found to metastasize to the extradural spinal location [12], so clinical signs of the metastasis may be apparent before clinical signs of the primary tumor [5]. However, in 53 dogs with intramedullary spinal neoplasia, $34 \%$ had secondary tumors, and only $44 \%$ of dogs with secondary tumors had myelopathic signs, because the remaining dogs was presented with primary organ dysfunction without myelopathic signs, including hemoabdomen, stranguria, and hematuria [14]. In the same study it was observed a high frequency of many kinds of carcinomas in the spinal cord, like transitional cell carcinoma, mammary, prostatic and pancreatic carcinoma [14].

Osteosarcoma is the most common primary bone tumor in dogs however, long bones are involved more often than flat bones [10]. In a study with 116 dogs with axial osteosarcoma, almost $17 \%$ of the cases were spinal [10]. Extradural osteosarcoma is a common extradural neoplasm $[5,9,11,15]$, causing proliferation as well as bone destruction, that are primary characteristic of osteosarcomas [4,15]. In studies about spinal tumors [6] or axial osteosarcoma [10,12], the prognosis for osteosarcomas is bad, and most dogs have a short survival, with or without surgical treatment.

Intramedullary spinal cord neoplasms are the least common of the three categories of spinal cord tumors [7], and in general are represented by primary glial tumors $[5,7]$ that were not diagnosed in the present study. However, we observed three cases of secondary spinal neoplasia, as carcinomas and hemangiosarcomas. In dogs, hemangiosarcoma have a propensity for intramedullary spinal cord involvement [5,6]. One intramedullary neoplasm was a anaplastic meningioma grade III, which in fact could be intradural-extramedullary initially and then because of the chronicity infiltrated into the spinal cord. Meningiomas generally show intraduralextramedullary localization and more commonly grow via compression and less commonly by infiltrating the nervous tissue [19]. However, there is a case report of intramedullary transitional mixed meningioma in the cervical spinal cord of a Boxer dog that probably had also infiltrated the spinal cord. [19].

The clinical course in relation to the onset of symptoms of neurological dysfunction, until death or euthanasia, ranged from 1-170 days, with an average 42 days of survival. After the diagnosis, the survival was short and this may be due to the fact that most dogs in this study already had more than one system affected, and consequently showed significant changes related to systemic metastases during the onset of the neurological symptoms. In one report of $37 \mathrm{dogs}$ with distint kind of spinal tumors, median survival of all dogs that survived 20 days following diagnosis was 240 days, but a total of $40 \%$ of these dogs, were euthanized or died within the first 20 days following diagnosis [15].

Only two dogs submitted to surgical treatment improved. The results observed after surgical resection of neoplasms vary with their location in relation to the spinal cord, neoplasm type, severity of the clinical signs, and possibility of removal with adequate margins without causing spinal instability $[5,13,18]$.

In conclusion, spinal neoplasia is an important differential diagnosis in dogs with localized or multifocal neurological signs. Secondary tumors were the majority of spinal cord neoplasms in the present study, so in dogs with spinal disorders, the veterinary practitioner should suspect spinal neoplasia, especially if there is a history of excision of neoplasms in other systems. Breeds that showed spinal neoplasms were mainly poodles and boxers, aged 5-10 years, and $67.85 \%$ of the cases were females. The thoracolumbar region was the most affected, predominantly with metastatic neoplasms, especially from mammary gland and skin neoplasms, and among the primary neoplasms, meningioma was the most common. As secondary spinal neoplasms were more common, the prognosis in most cases was poor. 
MANUFACTURERS

${ }^{1}$ Sanofi-Aventis. São Paulo, SP, Brazil.

${ }^{2}$ Biocare Medical. Concord, CA, USA.

${ }^{3}$ Zymed Laboratories. San Francisco, CA, USA

${ }^{4}$ Invitrogen Corporation. Carlsbad, CA, USA.
Declaration of interest. The authors report no conflicts of interest. The authors alone are responsible for the content and writing of paper.

\section{REFERENCES}

1 Adamo P.F., Cantile C. \& Steinberg H. 2003. Evaluation of progesterone and estrogen receptor expression in 15 meningiomas of dogs and cats. American Journal of Veterinary Research. 64(10): 1310-1318.

2 Adamo P.F., Forrest L. \& Dubielzig R. 2004. Canine and feline meningiomas: Diagnosis, treatment and prognosis. Compendium Continuing Education for Veterinarians. 26(12): 951-965.

3 Allett B. \& Hecht S. 2016. Magnetic resonance imaging findings in the spine of six dogs diagnosed with lymphoma. Veterinary Radiology \& Ultrasound. 57(2): 154-161.

4 Asano K., Kadosawa T., Mori T., Miyamoto T., Ueno H., Watanabe K. \& Fujinaga T. 2005. Ventilatory failure and successful management for a dog with severe cervical meningioma. The Journal of Veterinary Medical Science. 67(6): 599-602.

5 Bagley R.S. 2010. Spinal neoplasms in small animals. Veterinary Clinics of North America: Small Animal Practice. 40(5): 915-927.

6 Besalti O., Caliskan M., Can P., Vural S.A., Algin O. \& Ahlat O. 2016. Imaging and surgical outcomes of spinal tumors in $18 \mathrm{dogs}$ and one cat. Journal of Veterinary Science. 17(2): 225-234.

7 Braund K.G. 2003. Neoplasia of the Nervous System. In: Braund K.G. (Ed). Clinical Neurology in Small Animals. Localization, Diagnosis and Treatment. Ithaca:IVIS. Disponível em: http://www.ivis.org/special_books/Braund/braund26/ chapter_frm.asp?LA=1. [Accessed online December 2015].

8 Coomer A.R. \& Liptak J.M. 2008. Canine histiocytic diseases. Compendium Continuing Education for Veterinarians. 30(4): 202-216.

9 Da Costa R.C. 2008. Neoplasias do sistema nervoso. In: Daleck R.C., De Nardi A.B. \& Rodaski S. (Eds). Oncologia em cães e gatos. São Paulo: Roca, pp.411-436.

10 Heyman S.J., Diefenderfer D.L., Goldschmidt M.H. \& Newton C.D. 1992. Canine axial skeletal osteosarcoma. A retrospective study of 116 cases (1986 to 1989). Veterinary Surgery. 21(4): 304-310.

11 LeCouter R.A. \& Withrow S.J. 2007. Tumors of the nervous system. In: Stephen J. Withrow \& Vail D.M. (Eds). Withrow MacEwen's Small Animal Clinical Oncology. St. Louis: Elsevier, pp.659-685.

12 Mendonça F., Dória R., Freitas S., Pires M., Camargo L. \& Evêncio Neto J. 2008. Osteosarcoma vertebral em cão-relato de caso. Revista Clínica Veterinária. 76: 48-52.

13 North S.M. \& Banks T.A. 2009. Tumors of the Nervous System. In: Small Animal Oncology. An introduction. London: Elsevier, pp.243-251.

14 Pancotto T.E., Rossmeisl J.H., Zimmerman K., Robertson J.L. \& Were S.R. 2013. Intramedullary spinal cord neoplasia in 53 dogs (1990-2010): distribution, clinicopathologic characteristics, and clinical behavior. Journal of Veterinary Internal Medicine. 27(6): 1500-1508.

15 Petersen S.A., Sturges B.K., Dickinson P.J., Pollard R.E., Kass P.H., Kent M., Vernau K.M., Lecouteur R.A. \& Higgins R.J. 2008. Canine intraspinal meningiomas: imaging features, histopathologic classification, and long-term outcome in 34 dogs. Journal of Veterinary Internal Medicine. 22(4): 946-953.

16 Santos R.P., Fighera R.A., Beckmann D.V., Brum J.S., Ripplinger A., Dakir Neto P., Baumhardt R. \& Mazzanti A. 2012. Neoplasms affecting the central nervous system of dogs: 26 cases (2003-2011). Pesquisa Veterinária Brasileira. 32(2): 153-158.

17 Seguin B., Bagley R. \& Silver G. 2000. Diagnosis and treatment of spinal neoplasia in dogs and cats. Waltham Focus. 10: 4-9.

18 Suzano S., Amorin R. \& Qitzan J.2005. Oligodendroglioma medular em cão: relato de caso. Revista Clínica Veterinária. 58: 80-82.

19 Yalin E.E., Devecioglu Y., Demirutku A., Mutlu Z., Gurel A. \& Haktanir D. 2015. Intramedullary spinal cord meningioma in a Boxer: a case report. Veterinární medicina. 60(4): 222-226. 